\title{
Tai Chi, Qigong and the Treatment of Arthritis
}

\author{
Robert W McGee* \\ Department of Graduate and Professional Studies in Business, Fayetteville State University, USA \\ *Corresponding author: Robert W McGee, Department of Graduate and Professional Studies in Business, Fayetteville \\ State University, USA
}

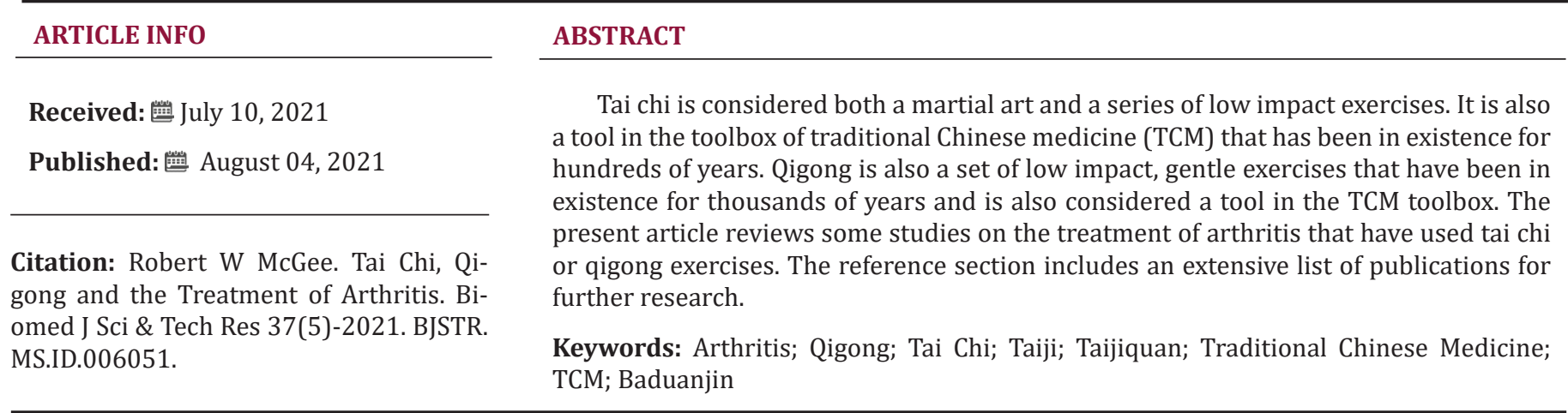

\section{Introduction}

Tai chi, also referred to as taiji or taijiquan, is considered both a martial art and a series of low impact exercises. It is also a tool in the toolbox of traditional Chinese medicine (TCM) that has been in existence for hundreds of years, although its philosophical roots go back thousands of years to Confucianism and Taoism [1]. There are five main styles of tai chi. Chen style is the oldest style and originated in the Chen village in China [2]. The second oldest style, and also the most popular style, is the Yang style [3]. Sun style [4] is the newest style of tai chi and has only been in existence for about 100 years. It is known for its high stances, which are especially suited for older people who have balance issues. Wu and Wu Hao [5] are the other main styles of tai chi, which contain elements of both the Yang and Chen styles. Although the styles all differ in some respects, they have more commonalities than differences.

Qigong [pronounced chee gong] is also a set of low impact, gentle exercises that have been in existence for thousands of years [6] and is also considered a tool in the TCM toolbox. Some practitioners consider qigong to be a subset of tai chi. Much has been written about qigong and qigong exercises [7-131]. The Chinese Health Qigong Association has produced nine DVDs that provide instruction on some of the more popular qigong exercise sets [132-140]. Several organizations have been formed to study various aspects of qigong [141-175].
Some publications focus on the application of tai chi or qigong for the treatment of arthritis [176-177]. Dr. Paul Lam, a Chinese physician based in Sydney, Australia, has developed several courses using the Sun style of tai chi to treat arthritis [178-179]. Numerous medical studies have been conducted on the effectiveness of tai chi and qigong on the treatment of anxiety [180-181, 227], arthritis [182-188], attention deficit [189], autism [190], back pain [191193], blood pressure [194-195], cancer [25, 196-215], chronic fatigue syndrome [216], cognitive performance [217-222], COPD [223], Covid-19 [71, 224-226], depression [227-238], dyspnea [239], fibromyalgia [240], frailty [241], geriatrics [242], heart disease [243-244], hypertension [245-246], immune system [247248], low back pain [249-250], mental health [251], neonatal intensive care [252], the nervous system [253-254], obesity [255], osteoporosis [256], pain management [257], Parkinson's disease [258-263], physical health [264-266], physical therapy [267], psychosis/psychological disorders [268-270], prostatic hyperplasia [271], pulmonary disease [272-276], quality of life [277-280], rheumatology [281], schizophrenia 282], sensory symptoms [283], sleep disorders [284], sports training [285], stress [286], stroke [287-290], substance abuse [291], and unilateral vocal fold paralysis [292]. A number of medical studies have examined the general effects of tai chi and qigong as well [293-301]. The present 
article reviews some studies on the treatment of arthritis that have used tai chi or qigong exercises.

\section{Methodology}

The PubMed.gov database [302] and the Europe PMC database [303] were used to find studies on tai chi, qigong and the treatment of arthritis, supplemented by internet searches.

\section{Findings}

Marks [304] reviewed, summarized, and synthesized the research involving the use of qigong exercises in the treatment of arthritis. He concluded that mind-body and relaxation techniques such as those practiced in China for centuries may be a useful intervention strategy for adults having various forms of arthritis. Numerous other studies have been conducted on the effectiveness of tai chi or qigong in general, as well as their effectiveness in the treatment of several specific diseases and ailments. The studies cited in this section examined the effectiveness of these tools of traditional Chinese medicine on various forms of arthritis.

\section{Osteoarthritis of the Knee}

Brosseau et al. [305] published clinical practice guidelines for the management of knee osteoarthritis after conducting a systematic study of controlled trials that used mind-body exercise programs for patients having osteoarthritis of the knee. The Ottawa panel of experts reached consensus on a number of issues. The main conclusion was that Sun-style tai chi exercises were effective in pain reduction and improvement of physical function and quality of life for individuals having osteoarthritis of the knee. Another Brosseau et al. study [306] reviewed 26 high-quality studies and identified various effective knee strengthening exercise programs. Other studies by the Ottawa panel examined the effects of various exercises on arthritis of the hip [307] and hand [308].

Li et al. [309] analyzed the biomechanical response of the meniscus and knee cartilage to the brush-knee and twist-step tai chi movement, and compared the results to walking and jogging. They found that the tai chi movement resulted in a greater increase in the range of motion, and that stress was more concentrated when walking and jogging than when doing the tai chi movement. The study concluded that tai chi may have a lower risk of knee joint injury than either walking or jogging.

You et al. [310] studied the effects of tai chi exercises on the improvement of walking function and posture control in elderly patients having osteoarthritis of the knee. After examining the results of 11 trials involving 603 participants, they concluded that the tai chi group had better performance in a six-minute walk test and the time up and go test than did the control group. The study concluded that tai chi could be an excellent way to improve walking function and posture control in elderly adults who have osteoarthritis of the knee. Zeng et al. [311] conducted a review of three random controlled trials (RCT) that indicated that baduanjin qigong exercises may have favorable effects on patients with osteoarthritis of the knee. Baduanjin patients had significantly better WOMAC (Western Ontario and McMaster Universities Arthritis Index) scores in some areas than did those of the control group. Significant differences occurred for pain $(\mathrm{p}<0.01)$, stiffness $(\mathrm{p}<0.01)$, and physical function $(\mathrm{p}<0.01)$.

Li et al. [312] conducted a systematic review and meta-analysis of 14 randomized control trials involving 815 patients to determine the effectiveness of traditional Chinese exercise (tai chi and qigong) on symptoms of osteoarthritis of the knee. Compared to the control group, the Chinese exercise group showed significant improvement in the Western Ontario and McMaster Universities Arthritis Index (WOMAC) and the Knee Injury and Osteoarthritis Outcome Score (KOOS) measures for pain ( $p<0.001)$, stiffness ( $p<0.001)$, and physical function score $(\mathrm{p}<0.001)$. The study concluded that tai chi and qigong may be effective for alleviating pain, relieving stiffness and improving physical function for patients suffering from osteoarthritis of the knee.

Xiao, Zhuang and Kang [313] examined the effects of wu qin xi (WQX), a set of qigong exercises, on physical functioning in elderly people with osteoarthritis of the knee using a random controlled trial (RCT). The study was a six-month follow-up of a RCT. The control group received physical therapy. While both groups had large reductions in activity limitations, pain and knee instability, the differences between the two groups were not statistically significant for the Timed Up and Go Test, 6-Minute Walk Test, knee extension strength and knee flexion strength, except for a higher Berg Balance Scale (BBS) score ( $p=0.029)$ and a lower Western Ontario and McMaster Universities Osteoarthritis Index pain score $(p=0.031)$ for the WQX group. The study concluded that while both WQX and conventional therapy were effective in reducing pain and activity limitations, and promoting muscle power and balance, WQX was significantly more effective in reducing pain and promoting balance than conventional therapy. Lee et al. [314] conducted a pilot, randomized, waiting list-controlled trial to determine whether tai chi exercises could be used to improve the quality of life for patients with osteoarthritis of the knee. The trial included forty-four elderly subjects who had arthritis of the knee. The exercise group took eight weeks of tai chi training, while the waiting list control group did not. Sessions were 60 minutes, two times per week. The training group had statistically significant improvements in quality of life $(\mathrm{p}<0.05)$ and the 6 -m walking test $(\mathrm{p}<0.01)$. Although the WOMAC scores for the training group were markedly improved, the differences were not statistically significant. The study concluded that tai chi exercises can have a beneficial effect on the quality of life and physical functioning in elderly patients who have osteoarthritis of the knee. 


\section{Osteoarthritis of the Hip}

Duan et al. [315] had 12 older, experienced men participate in a study that had them perform the brush knee and twist step tai chi move, along with normal walking. The study concluded that tai chi may result in a better improvement in the hip joint's range of motion and coordination of the neuromuscular system than just walking and is safe and suitable for patients with osteoarthritis of the hip. Zou et al. [316] examined the effect of tai chi practice on attenuating bone mineral density (BMD) loss. They examined the results of 20 randomized controlled trials having a total sample size of 1604. They found that tai chi produced significant benefits on BMD at the lumbar spine $(\mathrm{p}<0.0001)$, femur neck $(\mathrm{p}<0.00001)$, femur trochanter $(p=0.007)$, and total hip ( $p=0.003)$. They concluded that tai chi is effective in attenuating BMD.

Escalante et al. [317] summarized the evidence of 33 studies that evaluated the effectiveness of various exercise programs on pain for adults with lower limb osteoarthritis. They concluded that tai chi exercise programs achieved better results than strength programs, aerobic programs, hydrotherapy programs or mixed programs. Uhlig [318] found that tai chi and yoga are safe and can be used in the treatment of rheumatologic and musculoskeletal diseases such as osteoarthritis of the knee, hip, and hand, as well as rheumatoid arthritis.

\section{Ankylosing Spondylitis}

Xie et al. [319] conducted a randomized controlled trial of 60 patients to determine whether baduanjin, a set of 8 qigong exercises, would have a positive effect on the treatment of ankylosing spondylitis. Forty-six patients completed the study. The exercise group received 12 weeks of baduanjin training, while the other group received no treatment. Although the total Bath Ankylosing Spondylitis Disease Activity Index (BASDAI) scores between the two groups were not significantly different, the exercise group had reduced scores compared to the no-treatment group for fatigue $(p$ $=0.03)$, intensity $(\mathrm{p}=0.04)$, and duration of morning stiffness ( $\mathrm{p}=$ $0.01)$. The exercise group also had higher patient global assessment scores $(\mathrm{p}=0.04)$. The study concluded that the baduanjin exercises appeared to improve ankylosing spondylitis symptoms.

\section{Rheumatoid Arthritis}

Lee, Pittler and Ernst [320] conducted a systematic review of studies on the effect of tai chi on rheumatoid arthritis (RA) in 2007. They identified 45 potentially relevant studies. Some of the studies they found concluded some positive effects on the use of tai chi to relieve depression and mood of RA patients, while increasing the quality of life. Two studies found that tai chi did not result in any more pain reduction than what could be had with education, stretching exercises, and usual activity control. They concluded that the heterogeneity of the studies prevented any meaningful meta- analysis, and that the studies that were available at that time were of low methodological quality, concluding that whether tai chi was an effective treatment for rheumatoid arthritis remained unproven.

A more recent study by Imoto et al. [321] concluded that tai chi could be a low-cost alternative to other treatments of RA. Their study consisted of a review of three other studies. Those findings indicated that tai chi practice resulted in improvements in mood and depression. Although clinical improvement was achieved with regard to pain and disease pattern, the improvements were not statistically significant. A study by Wang [322] found that the practice of tai chi improved pain and functional status of adults with rheumatoid arthritis (RA). This study was a pilot randomized control trial consisting of twenty patients with functional class I or II RA who were randomly assigned either to tai chi or attention control. They received two sessions a week for 12 weeks. The tai chi group had significantly better results in the disability index $(\mathrm{p}=$ $0.01)$, and the depression index $(p=0.003)$. Similar improvement trends were observed in functional capacity and health-related quality of life and disease activity, while no adverse effects were observed.

Uhlig et al. [323] conducted a quantitative and qualitative study on the effects of tai chi on rheumatoid arthritis. They selected 15 patients from a rheumatology department of a hospital in Oslo, Norway, who performed tai chi exercises twice a week for 12 weeks, with a follow-up after 12 weeks. They found that the practice of tai chi resulted in improved lower-limb muscle function, both at the end of the 12-week sessions and in the twelve-week followups. Qualitatively, the patients had improved physical condition, confidence in moving, better balance and less pain, both during the exercise and in daily life. There was also a reduction in stress and increased body awareness. Patients practiced twelve movements in the Tai Chi for Arthritis program developed by Dr. Paul Lam, a Chinese physician living in Australia [178-179]. The Tai Chi for Arthritis program uses the Sun style [4] of tai chi.

\section{Concluding Comments}

The evidence is clear that tai chi and qigong can have beneficial effects on the treatment of various kinds of arthritis. However, there is room for more research to determine whether, and under what circumstances, these two tools of traditional Chinese medicine are more effective than alternative treatments. One area of research that has been neglected is to compare the various kinds of tai chi and qigong to determine whether one particular set of exercises might be more effective than others in the treatment of various diseases and ailments. For example, is baduanjin more effective in the treatment of $\mathrm{X}$ than other qigong sets? Or tai chi? Are some forms of tai chi or qigong more effective in the treatment of arthritis or other diseases than other forms? Is Yang style tai chi more effective in treating $\mathrm{X}$ than Sun style tai chi? 


\section{Conflict of Interests}

None.

\section{References}

1. (2021) Wikipedia. Tai chi.

2. (2021) Wikipedia. Chen-style taijiquan.

3. (2021) Wikipedia. Yang-style t’ai chi ch'uan.

4. (2021) Wikipedia. Sun-style t'ai chi ch'uan.

5. (2021) Wikipedia. Wu9Hao)-style t’ai chi ch'uan.

6. (2021) Wikipedia. History of Qigong.

7. Allen Katherine (2017) The Qigong Bible: The Definitive Guide to Energy Cultivation Exercise. Godsfield, UK,

8. Carson Patrick Emilio (2015) Liminality, Embodiment and the Six Healing Sounds of Qigong. Doctoral Dissertation. Florida State University.

9. Ce Jin, Hu Zhanggui, Jin Zhenghua (compilers) (1996) Practical Chinese Qigong for Home Health Care. Beijing: Foreign Languages Press.

10. Chang Pei-Shiun (2016) Physical and Psychological Effects of Qigong Exercise in Community-Dwelling Older Adults: An Exploratory Study. $\mathrm{PhD}$ dissertation. Yale University.

11. Chia Manta (1993) Healing Light of the Tao: Foundational Practices to Awaken Chi Energy. Rochester: Destiny Books.

12. Chia Mantak (2001) Cosmic Healing I: Cosmic Chi Kung. Chiang Mai, Thailand: Universal Tao Publications.

13. Chia Mantak, Dirk Oellibrandt (2001) Cosmic Healing II: Taoist Cosmology and Universal Healing Connections. Chiang Mai, Thailand: Universal Tao Publications.

14. Chou, Yvonne Wai Yi (2011) The Effects of Qigong on Reducing Stress, Anxiety and Enhancing Body-mind Wellbeing. PhD dissertation. Hong Kong Polytechnic University.

15. Cocke Mariad (2015) Altered Recovery Process after Fatiguing Exercise and Potential Benefits of Qigong in Patients with Fibromyalgia. Master of Science Thesis. University of Kansas.

16. Cohen Kenneth S (1999) The Way of Qigong: The Art and Science of Chinese Energy. Wellspring/Ballantine.

17. Davis Deborah (2008) Women's Qigong for Health \& Longevity. Boston \& London: Shambhala.

18. De Sousa, Liliana Filipa Martins (2012) Effects of Qigong on the Balance of Elderly People: A Feasibility Study. Master Thesis of Traditional Chinese Medicine. Universidade do Porto, Portugal.

19. Douglas Bill (1999) The Complete Idiot's Guide to T'ai Chi \& QiGong. New York: Alpha Books, a division of Macmillan General Reference.

20. Downey, Robert G (2018) Qigong for Internal Growth: Eight Brocades and Other Exercises to Develop Your Energy. Golden Flower Internal Arts.

21. Feng Tevia (2017) 8 Trigram Organ Qigong. White Tiger Qigong.

22. Feng Tevia (2017) 5 Element Qigong. Amazon.com.

23. Feng Tevia (2017) 5 Animal Qigong. White Tiger Qigong Publishing.

24. Fick Franklin (2011) Daoist Qi Gong in Ten Exercises. Shen Long Publishing.

25. Fick Franklin (2018) Immortal Fang's Longevity Qigong: Rubbing the Abdomen to Prevent Illness and Prolong Life. Amazon.com.

26. Fick Franklin (2018) 24 Section Brocade Qigong: Strengthen Body Longevity Method. Amazon.com.
27. Frantzis Bruce (2005) Opening the Energy Gates of Your Body: Qigong for Lifelong Health. Blue Snake Books.

28. Frantzis Bruce (2010) Dragon and Tiger Medical Qigong, Volume 1. Fairfax, CA: Energy Arts.

29. Frantzis Bruce (2014) Dragon and Tiger Medical Qigong Volume 2: Qi Cultivation Principles and Exercises. North Atlantic Books.

30. Frantzis Bruce, Paul Cavel (2020) Heaven and Earth Qigong Volume One: Heal Your Body and Awaken Your Chi. Amazon.com.

31. Friedman Suzanne (2006) Medical Qigong Exercise Prescriptions: A Self-Healing Guide for Patients \& Practitioners. Xlibris.

32. Friedman Suzanne (2006) The Yijing Medical Qigong System: A Daoist Medical I-Ching Approach to Healing. Xlibris.

33. Friedman Suzanne B (2009) Heal Yourself with Qigong. New Harbinger Publications.

34. Gaik Frances Veronica (2003) Merging East and West: A Preliminary Study Applying Spring Forest Qigong to Depression as an Alternative and Complementary Treatment. Psy.D. dissertation. Adler School of Professional Psychology.

35. Jahnke Roger (1997) The Healer Within. San Francisco: Harper.

36. Jahnke Roger (2002) The Healing Promise of Qi. New York: Contemporary Books, a division of McGraw-Hill.

37. Jianguo Zhang (1997) Qigong Exercise Therapy. Jinan, China: Shandong Science and Technology Press.

38. Jingwei li, Zhu Jianping (2014) The Illustrated Handbook of Chinese Qigong Forms from the Ancient Texts. London \& Philadelphia: Singing Dragon.

39. Johnson, Jerry Alan (2000) Chinese Medical Qigong Therapy: A Comprehensive Clinical Guide. Pacific Grove, CA: International Institute of Medical Qigong.

40. Johnson, Jerry Alan (2005) Chinese Medical Qigong Therapy, Vol. 1: Energetic Anatomy and Physiology. Pacific Grove, CA: International Institute of Medical Qigong.

41.Johnson, Jerry Alan (2005) Chinese Medical Qigong Therapy, Vol. 2: Pacific Grove, CA: International Institute of Medical Qigong.

42. Johnson Jerry Alan (2002) Chinese Medical Qigong Therapy, Vol. 3: Pacific Grove, CA: International Institute of Medical Qigong.

43. Johnson, Jerry Alan (2002) Chinese Medical Qigong Therapy, Vol. 4: Prescription Exercises and Meditations, Treatment of Internal Diseases, Pediatrics, Geriatrics, Gynecology, Neurology, and Energetic Psychology. Pacific Grove, CA: International Institute of Medical Qigong.

44. Johnson, Jerry Alan (2005) Chinese Medical Qigong Therapy, Vol. 5: An Energetic Approach to Oncology. Pacific Grove, CA: International Institute of Medical Qigong.

45. Kelly David (2017) Learn the Yi Jin Jing. SMA Bloggers. December 6.

46. Kelly Dave (2018) How to practice Liu Zi Jue - The six healing sounds. SMA Bloggers. May 4.

47. Kohn Livia (2008) Chinese Healing Exercises: The Tradition of Daoyin University of Hawaii Press.

48. Korahais Anthony (2014) Help! I Can't Decide What Qigong Exercises to Practice! Blog. April 9.

49. Korahais Anthony (2017) 13 Proven Benefits of Qigong \& Tai Chi. FlowingZen.com.

50. Korahais Anthony (2017) History of Qigong: The 5 Categories of Qi Cultivation. Bloc Post, February 14

51. Korahais Anthony (2017) Which Qigong Exercise Should You Practice for [Insert Problem]? Blog. October 10. 
52. Korahais Anthony (2019) Do You Need Specific Qigong Exercises for Specific Problems? Blog. May 7.

53. Korahais Anthony (2020) Blog Index.

54. Lam, Kam Chuen (2014) The Qigong Workbook for Anxiety. Oakland, CA: New Harbinger Publications.

55. Lei Yan (2009) Instant Health: The Shaolin Qigong Workout for Longevity. Yan Lei Press.

56. Li Zhi Cheng (2010) The Situation and Countermeasures of Health Qigong in Shanghai Older Age Groups of Community. Master's Degree Thesis. East China Normal University.

57. Liang Shou-yu, Wen-Ching Wu (2010) Qigong Empowerment: A Guide to Medical and Wushu Energy Cultivation. East Providence, RI: Way of the Dragon Publishing.

58. Liu Nan (2011) Effects of Health Qigong on Attention Resource Allocation and Automatic Processing in Middle-aged Women. Master's Degree Thesis. South China Normal University.

59. Liu Tianjun, Xiao Mei Qiang (2013) Chinese Medical Qigong. London \& Philadelphia: Singing Dragon.

60. Lopes, Lara de Jesus Teixeira (2015) The Acute Effect of "White Ball" Qigong in Perceptual Auditory Attention. Mestrado em Medicina Tradicional Chinesa, Universidade do Porto, Portugal.

61. Lu Yan (2011) Experimental Research of Expanding the Spare Exercises with Ba Duan Jin in the Group of Graduates - Take the Graduates of ECNU for Example. Master's Thesis. East China Normal University.

62. MacRitchie James (1993) Chi Kung: Cultivating Personal Energy. Element Books Ltd.

63. MacRitchie James (1997) The Chi Kung Way: Alive with Energy. Thorsons Publishers.

64. MacRitchie James (2003) Chi Kung: Energy for Life. Thorsons Publishers.

65. MacRitchie James (2018) The Eight Extraordinary Meridians Qigong Pdf document, 186 pages.

66. McGee Robert W (2020) Qigong: A Bibliography of Books and Other Materials, Fayetteville State University, Broadwell College of Business and Economics, Studies in the Economics of Qigong, No. 1, August 25.

67. McGee Robert W (2020) A Bibliography of Recent Medical Research on Qigong, Fayetteville State University, Broadwell College of Business and Economics, Studies in the Economics of Qigong, No. 2, August 31.

68. McGee Robert W (2020) Ba Duan Jin as a Treatment for Physical Ailments: A Bibliography of Recent Medical Research, Fayetteville State University, Broadwell College of Business and Economics, Studies in the Economics of Qigong, No. 3, August 31.

69. McGee Robert W (2020) Wu Qin Xi as a Treatment for Physical Ailments: A Bibliography of Recent Medical Research, Fayetteville State University, Broadwell College of Business and Economics, Studies in the Economics of Qigong, No. 4, August 31.

70. McGee, Robert W (2020) The Use of Yi Jin Jing to Treat Illness: A Summary of Three Studies, Fayetteville State University, Broadwell College of Business and Economics, Studies in the Economics of Qigong, No. 5, August 31

71. McGee Robert W (2020) Qigong and the Treatment and Prevention of COVID-19, Fayetteville State University, Broadwell College of Business and Economics, Studies in the Economics of Qigong, No. 6, September 4.

72. McGee, Robert W (2020) Qigong and the Treatment and Prevention of Cancer, Fayetteville State University, Broadwell College of Business and Economics, Studies in the Economics of Qigong, No. 7, September 14.

73. McGee, Robert W (2021) Tai Chi, Qigong and Transgender Health. Fayetteville State University, Broadwell College of Business and Economics, Studies in the Economics of Tai Chi and Qigong, No. 8, April 16.
74. McGee, Robert W (2021) Tai Chi, Qigong and the Treatment of Disease. Biomedical Journal of Scientific \& Technical Research 34(2): $26627-$ 26633 (2021).

75. McGee, Robert W (2021) Tai Chi, Qigong and the Treatment of Cancer Biomedical Journal of Scientific \& Technical Research, 34(5): 27,17327,182 (2021).

76. McGee, Robert W (2021) The Use of Yi Jin Jing to Treat Illness: A Summary of Three Studies. Academia Letters, Article 547, April 2021.

77. McGee, Robert W (2021) Tai Chi, Qigong and the Treatment of Depression and Anxiety. Biomedical Journal of Scientific \& Technical Research 36(2): 28350-28354 (2021)

78. Mitchell, Damo (2013) Heavenly Streams: Meridian Theory in Nei Gong. London \& Philadelphia: Singing Dragon.

79. Mitchell, Damo (2019) Yi Jin Jing Principles. Scholar Sage. June 6.

80. Moon, Sanghee (2019) Effects of a Mind-Body Exercise on Non-motor Symptoms and Inflammatory Biomarkers in Parkinson's Disease. PhD dissertation. University of Kansas.

81. Morris, Kim Lisa Chou (1998) On the Chinese Spiritual Tradition of Qigong in Toronto as a Site for Negotiating Identity. Master of Fine Arts Thesis. York University (Canada).

82. Munro John (2013) Qigong: Foundation Practices. Infosource Ltd.

83. Munro John (2017) Enter the Flow: Mindful Movement Qigong. Amazon. com

84. Munro John (2017) Waking the Qi: An Introduction to Qigong. Amazon. com.

85. Munro John (2017) Between Heaven and Earth: Qigong for the Eight Extraordinary Meridians. Amazon.com.

86. Murakawa Haruhiko (2002) Phenomenology of the Experience of Qigong: A Preliminary Research Design for the Intentional Bodily Practices. PhD dissertation. California Institute of Integral Studies.

87. Palmer David A (2007) Qigong Fever. Columbia University Press.

88. Pangen Qigong.

89. Ramlal Derek (2007) Qigong and the Modernization of China. Doctoral Dissertation (D.A.), Saint John's University (New York).

90. Ross David-Dorian (2014) Essentials of Tai Chi and Qigong: Course Guidebook. Chantilly, VA: The Great Courses.

91. Sarmento, Caio Vinicius Messias (2019) Changes in Immune Response to High Intensity Exercise in Patients Fibromyalgia before and after a Mind-Body Intervention. PhD dissertation. University of Kansas.

92. Schoeninger, Kevin D (2006) Learn Qigong Meditation: A Seven-Week Home-Study Course. Lafayette, CO: The Power of Practice.

93. Scholar Sage (2020) Many articles.

94. Scientific Premium Company - USA (2009) Master Secrets of Qigong \& Chi Power.

95. Shanghai Qi Gong Institute (2013) Discovering Chinese Philosophy through Qi Gong Body Awareness. Shanghai: Shanghai Qi Gong Institute.

96. Sveund Jennifer (2017) The Experience of Qigong Among Women Cancer Survivors. Antioch University. Dissertations \& Theses. 378.

97. Taiji Forum (2020) 5 Animal Qigong. Taiping Institute. 2020. Wu Qin Xi.

98. Taiji Yang Sheng Zhang Tai Chi Stick (2020) Tundeworld.

99. Taiping Institute (2020) Wu Qin Xi.

100. Tapajna Mark (2006) An Examination of Medical Qigong: Its Practice, Application, and Potential Value for a Western Society. PhD dissertation. Cleveland State University. 
101. (2021) Tundeworld Yi Jin Jing.

102. (2021) Tundeworld. Many articles.

103. Wang Chunyun (2012) Improving Health among Elementary School Children: A Comparison of Aerobic and Mind-Body Exercise. PhD dissertation. Indiana University.

104. Wang, Yong Tai, Guoyuan Huang, Gloria Duke, Yi Yang (2017) Tai Chi, Yoga, and Qigong as Mind-Body Exercises. Hindawi Publishing Corporation.

105. (2021) Wikipedia. Qigong.

106. (2021) Wikipedia. 2021. History of Qigong.

107. (2021) Wikipedia. Baduanjin qigong.

108. (2021) Wikipedia. Liu Zi Jue.

109. (2021) Wikipedia. Five Animals.

110. (2021) Wikipedia. Yi Jin Jing.

111. Wu Zhongxian (2011) Chinese Shamanic Cosmic Orbit Qigong. London \& Philadelphia: Singing Dragon.

112. Wu Zhongxian (2012) Fire Dragon Meridian Qigong: Essential NeiGong for Health and Spiritual Transformation. London \& Philadelphia: Singing Dragon.

113. Wu Zhongxian (2012) Vital Breath of the Dao: Chinese Shamanic Tiger Qigong - Laohu Gong. London \& Philadelphia: Singing Dragon.

114. Yang Jwing-Ming (1997) The Root of Chinese Qigong: Secrets of Health, Longevity \& Enlightenment. Roslindale, MA: YMAA Publication Center.

115. Yang Jwing-Ming (1997) Eight Simple Qigong Exercises for Health: The Eight Pieces of Brocade. Roslindale, MA: YMAA Publication Center

116. Yang Jwing-Ming (1998) Qigong for Health and Martial Arts: Exercises \& Meditation. Boston, MA: YMAA Publication Center.

117. Yang Jwing-Ming (2000) Qigong: The Secret of Youth. Boston, MA YMAA Publication Center.

118. Yang Jwing-Ming (2003) Qigong Meditation: Embryonic Breathing. Boston, MA: YMAA Publication Center.

119. Yang Jwing-Ming (2015) Tai Chi Qigong: The Internal Foundation of Tai Chi Chuan. Roslindale, MA: YMAA Publication Center.

120. Yang Jwing-Ming (2016) Meridian Qigong Exercises: Combining Qigong, Yoga \& Acupressure. Roslindale, MA: YMAA Publication Center

121. Yang Jwing-Ming (2016) The Essence of Shaolin White Crane: Martial Power and Qigong. Roslindale, MA: YMAA Publication Center.

122. Yang Jwing-Ming (2016) Qigong Meditation: Embryonic Breathing. Roslindale, MA: YMAA Publication Center.

123. Yang Jwing-Ming (2016) Qigong Meditation: Small Circulation Roslindale, MA: YMAA Publication Center.

124. Yang, Jwing-Ming (2017) The Pain-Free Back: Gentle Qigong Movements for Healing and Prevention. Roslindale, MA: YMAA Publication Center.

125. Yang Jwing-Ming (2018) The Dao De Jing: A Qigong Interpretation. Roslindale, MA: YMAA Publication Center.

126. Yang Jwing-Ming (2019) Tai Chi Ball Qigong: For Health and Martial Arts Roslindale, MA: YMAA Publication Center.

127. Yi Jin Jing (2020) Tundeworld.

128. Yongsheng Bi, Sun Hua, Guo Yi, Cao Zhenhua, Zhang Mingqin, Zhang Bohua (1988) Chinese Qigong. Publishing House of the Shanghai College of Traditional Chinese Medicine.

129. Yost Terri Lynn (2011) Qigong as a Novel Intervention in Service Members with Mild Traumatic Brain Industry. PhD dissertation. University of Virginia.
130. Zhao, Jin Xiang (1997) Chinese Soaring Crane Qigong. Corvallis, OR: Qigong Association of America.

131. Zhineng Qigong Science Worldwide.

132. Liu Zi Jue (2007) Chinese Health Qigong Association. Beijing: Foreign Languages Press.

133. Ba Duan Jin (2008) Chinese Health Qigong Association. Beijing: Foreign Languages Press.

134. Wu Qin Xi (2008) Chinese Health Qigong Association. Beijing: Foreign Languages Press.

135. Yi Jin Jing (2009) Chinese Health Qigong Association. Beijing: Foreign Languages Press.

136. Da Wu (2014) Chinese Health Qigong Association. Beijing: Foreign Languages Press.

137. Shi Er Duan Jin (2014) Chinese Health Qigong Association. Beijing: Foreign Languages Press.

138. Daoyin Yangsheng Gong Shi Er Fa (2014) Chinese Health Qigong Association. Beijing: Foreign Languages Press.

139. Mawanhdui Daoyin Shu (2014) Chinese Health Qigong Association. Beijing: Foreign Languages Press.

140. Taiji Yangsheng Zhang (2014) Chinese Health Qigong Association. Beijing: Foreign Languages Press.

141. American Academy for Medical Qigong.

142. American Qigong Association.

143. American Tai Chi and Qigong Association.

144. British Health Qigong Association.

145. Center for Taiji \& Qigong Studies.

146. The Chi Center.

147. Energy Arts.

148. European Sports Qigong Association.

149. Flowing Zen Qigong \& Tai Chi.

150. Holden Qigong.

151. Hong Kong Tai Chi \& Qigong.

152. The Institute for Rehabilitative Qigong \& Tai Chi.

153. Institute of Integral Qigong and Tai Chi.

154. Instituto Qigong Barcelona.

155. International Health Qigong Federation.

156. International Institute of Medical Qigong.

157. International Medical Qigong College.

158. International Medical Tai Chi and Qigong Association.

159. International Qi Gong Association.

160. Lin Housheng's Qigong.

161. National Qigong Association.

162. Qi Journal.

163. Qigong Awareness.

164. Qigong and Daoist Training Center.

165. Qigong Global Summit.

166. Qigong Institute.

167. The Qigong Research Society. 


\section{Robert Peng.}

169. Spring Forest Qigong.

170. Tai Chi Healthways.

171. Tai Chi, Qigong \& Feng Shui Institute.

172. Tse Qigong Centre.

173. United States Health Qigong Association.

174. World Tai Chi \& Qigong Day.

175. Yoqi Yoga \& Qigong.

176. Yang Jwing-Ming (2005) Arthritis Relief: Chinese Qigong for Healing and Prevention. Roslindale, MA: YMAA Publication Center.

177. Yang Jwing-Ming (2017) Pain-Free Joints: Simple Qigong Movements for Arthritis Healing and Prevention. Roslindale, MA: YMAA Publication Center.

178. (2021) Tai Chi for Health Institute.

179. (2021) Tai Chi for Arthritis Video. Dr. Paul Lam. Free Lesson and Introduction. Tai Chi for Health Institute.

180. Li Z, Liu S, Wang L, Smith L (2019) Mind-Body Exercise for Anxiety and Depression in COPD Patients: A Systematic Review and Meta-Analysis. International journal of environmental research and public health, 17(1): 22

181. Liu F, Cui J, Liu X, Chen KW, Chen X, Li R (2020) The effect of tai chi and Qigong exercise on depression and anxiety of individuals with substance use disorders: a systematic review and meta-analysis. BMC complementary medicine and therapies 20(1): 161.

182. Chen X, Cui J, Li R, Norton R, Park J, Kong J, Yeung A (2019) Dao Yin (a.k.a. Qigong): Origin, Development, Potential Mechanisms, and Clinical Applications. Evidence-based complementary and alternative medicine: eCAM, 3705120

183. Liu B, Fan Z, Wang Z, Li M, Lu T (2020) The efficacy and safety of Health Qigong for ankylosing spondylitis: Protocol for a systematic review and meta-analysis. Medicine 99(3): e18734.

184. Marks R (2019) Qigong and Musculoskeletal Pain. Current rheumatology reports 21(11): 59.

185. Xie Y, Guo F, Lu Y, Guo Y, Wei G, et al. (2019) A 12-week Baduanjin Qigong exercise improves symptoms of ankylosing spondylitis: A randomized controlled trial. Complementary therapies in clinical practice, 36: 113-119.

186. Ye J, Simpson MW, Liu Y, Lin W, Zhong W, et al. (2020) The Effects of Baduanjin Qigong on Postural Stability, Proprioception, and Symptoms of Patients with Knee Osteoarthritis: A Randomized Controlled Trial. Frontiers in medicine 6: 307

187. Ye J, Zheng Q, Zou L, Yu Q, Veronese N, Grabovac I, et al. (2020) Mindful Exercise (Baduanjin) as an Adjuvant Treatment for Older Adults (60 Years Old and Over) of Knee Osteoarthritis: A Randomized Controlled Trial. Evidence-based complementary and alternative medicine: eCAM, 2020, 9869161.

188. Zeng ZP, Liu YB, Fang J, Liu Y, Luo J, (2020) Effects of Baduanjin exercise for knee osteoarthritis: A systematic review and meta-analysis. Complementary therapies in medicine 48: 102279.

189. Duarte L, Gonçalves M, Mendes P, Matos LC, Greten HJ, et al. (2020) Can Qigong improve attention in adolescents? A prospective randomised controlled trial. Journal of bodywork and movement therapies 24(1): $175-181$.

190. Rodrigues JM, Mestre M, Fredes LI (2019) Qigong in the treatment of children with autism spectrum disorder: A systematic review. J Integr Med 17(4): 250-260
191. Chen X, Cui J, Li R, Norton R, Park J, et al. (2019) Dao Yin (a.k.a. Qigong): Origin, Development, Potential Mechanisms, and Clinical Applications. Evidence-based complementary and alternative medicine: eCAM, 2019: 3705120

192. Park J, Krause-Parello CA, Barnes CM (2020) A Narrative Review of Movement-Based Mind-Body Interventions: Effects of Yoga, Tai Chi, and Qigong for Back Pain Patients. Holistic nursing practice 34(1): 3-23.

193. Zou L, Zhang Y, Yang L, Loprinzi PD, Yeung AS, et al. (2019) Are Mindful Exercises Safe and Beneficial for Treating Chronic Lower Back Pain? A Systematic Review and Meta-Analysis of Randomized Controlled Trials. Journal of clinical medicine 8(5): 628.

194. Liu D, Yi L, Sheng M, Wang G, Zou Y (2020) The Efficacy of Tai Chi and Qigong Exercises on Blood Pressure and Blood Levels of Nitric Oxide and Endothelin-1 in Patients with Essential Hypertension: A Systematic Review and Meta-Analysis of Randomized Controlled Trials. Evidence-based complementary and alternative medicine: eCAM, 2020: 3267971.

195. Park JE, Yang CS, Jung SY, Kim AR, Lee M, et al. (2019) Exploring the effects of expert-led qigong and self-practice qigong on blood pressure. Integrative medicine research 8(4): 271-272.

196. Arring NM, Barton DL, Brooks T, Zick SM (2019) Integrative Therapies for Cancer-Related Fatigue. Cancer journal (Sudbury, Mass.) 25(5): 349-356.

197. Campbell KL, Zadravec K, Bland KA, Chesley E, Wolf F, et al. (2020) The Effect of Exercise on Cancer-Related Cognitive Impairment and Applications for Physical Therapy: Systematic Review of Randomized Controlled Trials. Physical therapy 100(3): 523-542.

198. Chang PS, Knobf T (2019) Qigong Exercise and Tai Chi in Cancer Care. Asia-Pacific journal of oncology nursing 6(4): 315-317.

199. Cheng TC, Lee YH, Mar CL, Huang WT, Chang YP (2020) The Health Promoting Mindfulness or Qigong Educational Programs for Beneficial Lifestyle Changes of Cancer Survivors. Journal of cancer education: the official journal of the American Association for Cancer Education, 35(4): 743-750

200. Deng G (2019) Integrative Medicine Therapies for Pain Management in Cancer Patients. Cancer journal (Sudbury, Mass.) 25(5): 343-348.

201. Ford CG, Vowles, KE, Smith BW, Kinney AY (2020) Mindfulness and Meditative Movement Interventions for Men Living with Cancer: A Meta-analysis. Annals of behavioral medicine: a publication of the Society of Behavioral Medicine 54(5): 360-373.

202. Garland SN, Mahon K,Irwin MR (2019) Integrative Approaches for Sleep Health in Cancer Survivors. Cancer journal (Sudbury, Mass.) 25(5): 337-342.

203. Larkey L, Kim W, James D, Kishida M, Vizcaino M, et al. (2020) MindBody and Psychosocial Interventions May Similarly Affect Heart Rate Variability Patterns in Cancer Recovery: Implications for a Mechanism of Symptom Improvement. Integrative cancer therapies 19: 1534735420949677

204. Li F, Wang C (2020) A Good Guy Again: Biosociality in a Cancer Selfhelp Organization. Medical anthropology: 1-14.

205. Lin WF, Zhong MF, Zhou QH, Zhang YR, Wang H, et al. (2019) Efficacy of complementary and integrative medicine on health-related quality of life in cancer patients: a systematic review and meta-analysis. Cancer management and research 11: 6663-6680.

206. Maindet C, Burnod A, Minello C, George B, Allano G, et al. (2019) Strategies of complementary and integrative therapies in cancerrelated pain-attaining exhaustive cancer pain management. Supportive care in cancer : official journal of the Multinational Association of Supportive Care in Cancer 27(8): 3119-3132. 
207. Oberoi D, Piedalue KL, Pirbhai H, Guirguis S, Santa Mina D, et al. (2020) Factors related to dropout in integrative oncology clinical trials: interim analysis of an ongoing comparative effectiveness trial of mindfulness-based cancer recovery and Tai chi/Qigong for cancer health (The MATCH study). BMC research notes 13(1): 342.

208. Sowada KM (2019) Qigong: Benefits for Survivors Coping with CancerRelated Fatigue. Clinical journal of oncology nursing 23(5): 465-469.

209. Wang FF, Yuan Y, Song YJ, Wu YQ, He Y, et al. (2020) Exercise or sport activities for patients with cancer: A protocol for overview of systematic reviews and meta-analyses. Medicine, 99(20): e20084.

210. Kreutz C, Schmidt ME, Steindorf, K (2019) Effects of physical and mind-body exercise on sleep problems during and after breast cancer treatment: a systematic review and meta-analysis. Breast cancer research and treatment 176(1): 1-15.

211. Osypiuk K, Ligibel J, Giobbie-Hurder A, Vergara-Diaz G, Bonato P, et al. (2020) Qigong Mind-Body Exercise as a Biopsychosocial Therapy for Persistent Post-Surgical Pain in Breast Cancer: A Pilot Study. Integrative cancer therapies 19: 1534735419893766.

212. Zhang Q, Gao X, Liu S, Yu L, Zhu J, et al. (2020) Therapies for cognitive impairment in breast cancer survivors treated with chemotherapy: A protocol for systematic review. Medicine 99(19): e20092.

213. Henshall CL, Allin L, Aveyard H (2019) A Systematic Review and Narrative Synthesis to Explore the Effectiveness of Exercise-Based Interventions in Improving Fatigue, Dyspnea, and Depression in Lung Cancer Survivors. Cancer nursing 42(4): 295-306.

214. Kinney AY, Blair CK, Guest DD, Ani JK, Harding EM, et al. (2019) Biobehavioral effects of Tai Chi Qigong in men with prostate cancer: Study design of a three-arm randomized clinical trial. Contemporary clinical trials communications 16: 100431

215. Zuniga KB, Chan JM, Ryan CJ, Kenfield SA (2020) Diet and lifestyle considerations for patients with prostate cancer. Urologic oncology 38(3): 105-117.

216. Xie F, Guan C, Cheng Z, Yao F, You Y (2020). Effects of the prolong life with nine turn method (Yan Nian Jiu Zhuan) Qigong on patients with chronic fatigue syndrome: study protocol for a randomized controlled trial. Annals of palliative medicine, apm: 19-461.

217. Campbell, K L, Zadravec K, Bland KA, Chesley E, Wolf F, et al.. (2020) The Effect of Exercise on Cancer-Related Cognitive Impairment and Applications for Physical Therapy: Systematic Review of Randomized Controlled Trials. Physical therapy 100(3): 523-542.

218. Chan J, Deng K, Wu J, Yan JH (2019) Effects of Meditation and MindBody Exercises on Older Adults' Cognitive Performance: A Metaanalysis. The Gerontologist 59(6): e782-e790.

219. Ibañez GE, Fennie K, Larkey L (2019) Qigong as a promising mindbody exercise for cognitive functioning: Letter to Editor. Journal of the American Geriatrics Society 67(7): 1533-1534

220. Wu C, Tang C (2019) Reply to: Qigong as a Promising Mind-Body Exercise for Cognitive Functioning. Journal of the American Geriatrics Society 67(7): 1534-1535.

221. Zhang Q Gao X, Liu S, Yu L, Zhu J, et al. (2020) Therapies for cognitive impairment in breast cancer survivors treated with chemotherapy: A protocol for systematic review. Medicine 99(19): e20092.

222. ZouL, Loprinzi PD, Yeung AS, Zeng N, Huang T (2019). The Beneficial Effects of Mind-Body Exercises for People with Mild Cognitive Impairment: a Systematic Review with Meta-analysis. Archives of physical medicine and rehabilitation 100(8): 1556-1573.

223. Li Z, Liu S, Wang L, Smith L (2019) Mind-Body Exercise for Anxiety and Depression in COPD Patients: A Systematic Review and Meta-Analysis. International journal of environmental research and public health 17(1): 22 .
224. Chen JM, Wang ZY, Chen YJ, Ni J (2020) The Application of Eight-Segment Pulmonary Rehabilitation Exercise in People with Coronavirus Disease 2019. Frontiers in physiology11: 646.

225. Feng F, Tuchman S, Denninger JW, Fricchione GL, Yeung A (2020) Qigong for the Prevention, Treatment, and Rehabilitation of COVID-19 Infection in Older Adults. The American journal of geriatric psychiatry: official journal of the American Association for Geriatric Psychiatry, 28(8): 812-819.

226. Zhang S, Zhu Q, Zhan C, Cheng W, Mingfang X, et al. (2020) Acupressure therapy and Liu Zi Jue Qigong for pulmonary function and quality of life in patients with severe novel coronavirus pneumonia (COVID-19): a study protocol for a randomized controlled trial. Trials 21(1): 751.

227. McGee, Robert W (2021) Tai Chi, Qigong and the Treatment of Depression and Anxiety. Biomedical Journal of Scientific \& Technical Research 36(2): 28350-28354 (2021).

228. Gill BK, Cant R, Lam L, Cooper S, Lou V (2020) Non-pharmacological depression therapies for older Chinese adults: A systematic review \& meta-analysis. Archives of gerontology and geriatrics 88: 104037.

229. Gouw V, Jiang Y, Seah B, He H, Hong J, Wang W (2019) Effectiveness of internal Qigong on quality of life, depressive symptoms and selfefficacy among community-dwelling older adults with chronic disease: A systematic review and meta-analysis. International journal of nursing studies 99: 103378

230. Henshall CL, Allin L, Aveyard H (2019) A Systematic Review and Narrative Synthesis to Explore the Effectiveness of Exercise-Based Interventions in Improving Fatigue, Dyspnea, and Depression in Lung Cancer Survivors. Cancer nursing 42(4): 295-306.

231. Jin X, Wang L, Liu S, Zhu L, Loprinzi, PD, et al. (2019. The Impact of Mind-body Exercises on Motor Function, Depressive Symptoms, and Quality of Life in Parkinson's Disease: A Systematic Review and Metaanalysis. International journal of environmental research and public health 17(1): 31 .

232. Lee P, Cai S, Lu EY, Ng B, Jensen MP, et al. (2020) Qigong Reduces Depressive Symptoms of Taiwanese Elderly with Chronic Physical Illness: A Randomized Controlled Trial. Journal of alternative and complementary medicine (New York, N.Y.) 26(1): 76-78.

233. Lee P, Cai S, Lu EY, Ng B, Jensen MP, et al. (2020) Qigong Reduces Depressive Symptoms of Taiwanese Elderly with Chronic Physical Illness: A Randomized Controlled Trial. Journal of alternative and complementary medicine (New York, N.Y.) 26(1): 76-78.

234. Li Z, Liu S, Wang L, Smith L (2019). Mind-Body Exercise for Anxiety and Depression in COPD Patients: A Systematic Review and Meta-Analysis. International journal of environmental research and public health 17(1): 22 .

235. Liu F, Cui J, Liu X, Chen KW, Chen X, et al. (2020) The effect of tai chi and Qigong exercise on depression and anxiety of individuals with substance use disorders: a systematic review and meta-analysis. BMC complementary medicine and therapies 20(1): 161.

236. Lu EY, Lee P, Cai S, So W, Ng B, et al. (2020) Qigong for the treatment of depressive symptoms: Preliminary evidence of neurobiological mechanisms. International journal of geriatric psychiatry, 10.1002/ gps.5380.

237. So WWY, Cai S, Yau SY, Tsang HWH (2019) The Neurophysiological and Psychological Mechanisms of Qigong as a Treatment for Depression: A Systematic Review and Meta-Analysis. Front Psychiatry.10: 820.

238. Zaprutko T, Göder R, Rybakowski F, Kus K, Kopciuch D, etal. (2020) Nonpharmacological treatments of inpatients with major depression - The case of Polish (Poznan) and German (Kiel) hospital. Complementary therapies in clinical practice, 39, 101129.

239. Henshall CL, Allin L, Aveyard H (2019) A Systematic Review and Narrative Synthesis to Explore the Effectiveness of Exercise-Based 
Interventions in Improving Fatigue, Dyspnea, and Depression in Lung Cancer Survivors. Cancer nursing 42(4): 295-306.

240. Sarmento C, Moon S, Pfeifer T, Smirnova IV, Colgrove Y, et al. (2020) The therapeutic efficacy of Qigong exercise on the main symptoms of fibromyalgia: A pilot randomized clinical trial. Integrative medicine research 9(4): 100416.

241. Liu X, Seah J, Pang B, Tsao MA, Gu F, et al. (2020) A single-arm feasibility study of community-delivered Baduanjin (Qigong practice of the eight Brocades) training for frail older adults. Pilot and feasibility studies 6: 105.

242. Liu X, Seah J, Pang B, Tsao MA, Gu F, et al. (2020) A single-arm feasibility study of community-delivered Baduanjin (Qigong practice of the eight Brocades) training for frail older adults. Pilot and feasibility studies 6: 105 .

243. Chen X, Cui J, Li R, Norton R, Park J, et al. (2019) Dao Yin (a.k.a. Qigong): Origin, Development, Potential Mechanisms, and Clinical Applications. Evidence-based complementary and alternative medicine : eCAM 2019: 3705120.

244. Mao S, Zhang X, Chen M, Wang C, Chen Q et al. (2020) Beneficial Effects of Baduanjin Exercise on Left Ventricular Remodelling in Patients after Acute Myocardial Infarction: an Exploratory Clinical Trial and Proteomic Analysis. Cardiovascular drugs and therapy,

245. Dong DG, Yu ZD, Yu ZS (2020) Zhongguo ying yong sheng li xue za zhi = Zhongguo yingyong shenglixue zazhi $=$ Chinese journal of applied physiology 36(2): 157-160.

246. Liu D, Yi L, Sheng M, Wang G, Zou Y (2020) The Efficacy of Tai Chi and Qigong Exercises on Blood Pressure and Blood Levels of Nitric Oxide and Endothelin-1 in Patients with Essential Hypertension: A Systematic Review and Meta-Analysis of Randomized Controlled Trials. Evidence-based complementary and alternative medicine: eCAM 2020, 3267971.

247. Chen X, Cui J, Li R, Norton R, Park J, et al. (2019) Dao Yin (a.k.a. Qigong): Origin, Development, Potential Mechanisms, and Clinical Applications Evidence-based complementary and alternative medicine: eCAM, 2019:3705120.

248. Oh B, Bae K, Lamoury G, Eade T, Boyle F, et al. (2020) The Effects of Tai Chi and Qigong on Immune Responses: A Systematic Review and MetaAnalysis. Medicines (Basel, Switzerland) 7(7): 39.

249. Chen X, Cui J, Li R, Norton R, Park J, et al. (2019) Dao Yin (a.k.a. Qigong): Origin, Development, Potential Mechanisms, and Clinical Applications Evidence-based complementary and alternative medicine: eCAM, 2019: 3705120.

250. Zou L, Zhang Y, Yang L, Loprinzi, PD, Yeung AS, et al. (2019) Are Mindful Exercises Safe and Beneficial for Treating Chronic Lower Back Pain? A Systematic Review and Meta-Analysis of Randomized Controlled Trials. Journal of clinical medicine, 8(5): 628.

251. Guo C, Xiang G, Xie L, Liu Z, Zhang X, et al. (2020) Effects of Tai Chi training on the physical and mental health status in patients with chronic obstructive pulmonary disease: a systematic review and metaanalysis. Journal of thoracic disease 12(3): 504-521.

252. Johnson Rolfes J, Christensen K, Gershan LA (2020) Acceptance of Traditional Chinese Medicine in the Neonatal Intensive Care Unit: A Launching Point. Global advances in health and medicine 9: 2164956120924644

253. Chen X, Cui J, Li R, Norton R, Park J, et al. (2019). Dao Yin (a.k.a. Qigong): Origin, Development, Potential Mechanisms, and Clinical Applications. Evidence-based complementary and alternative medicine: eCAM, 2019:3705120.

254. Chin MS, Kales SN (2019) Understanding mind-body disciplines: A pilot study of paced breathing and dynamic muscle contraction on autonomic nervous system reactivity. Stress and health: journal of the International Society for the Investigation of Stress 35(4): 542-548.
255. Lin WY, Chan CC, Liu YL, Yang AC, Tsai SJ, et al. (2019) Performing different kinds of physical exercise differentially attenuates the genetic effects on obesity measures: Evidence from 18,424 Taiwan Biobank participants. PLoS genetics 15(8): e1008277.

256. Liu M, Liu D, Hong P, Qiu X, Chen Q (2020) The effect of Qigong Wuqinxi for osteopenia and primary osteoporosis: A protocol for systematic review and meta analysis. Medicine 99(21): e20379.

257. Tang SK, Tse M, Leung SF, Fotis T (2019) The effectiveness, suitability, and sustainability of non-pharmacological methods of managing pain in community-dwelling older adults: a systematic review. BMC public health 19(1): 1488.

258. Chen S, Zhang Y, Wang YT, Liu X, Song W, et al. (2020) The effect of Qigong-based therapy on patients with Parkinson's disease: a systematic review and meta-analysis. Clinical rehabilitation 269215520946695. Advance online publication.

259. Fidan O, Seyyar GK, Aras B, Colak E, Aras O (2019) The effect of Tai Chi and Qigong on health-related quality of life in Parkinson's disease: a systematic review and meta-analysis of systematic reviews. International journal of rehabilitation research. Internationale Zeitschrift fur Rehabilitations for schung. Revue internationale de recherches de readaptation 42(3): 196-204.

260. Jin X, Wang L, Liu S, Zhu L, Loprinzi PD, Fan X (2019) The Impact of Mind-body Exercises on Motor Function, Depressive Symptoms, and Quality of Life in Parkinson's Disease: A Systematic Review and Metaanalysis. International journal of environmental research and public health 17(1): 31.

261. Li Z, Zhuang J, Jiang Y, Xiao G, Jie K, et al. (2019) Study protocol for a single-blind randomised controlled trial to evaluate the clinical effects of an Integrated Qigong exercise intervention on freezing of gait in Parkinson's disease. BMJ open 9(9): e028869.

262. Moon S, Sarmento C, Steinbacher M, Smirnova IV, Colgrove, et al. (2020) Can Qigong improve non-motor symptoms in people with Parkinson's disease - A pilot randomized controlled trial?. Complementary therapies in clinical practice 39: 101169.

263. Tang L, Fang Y, Yin J (2019) The effects of exercise interventions on Parkinson's disease: A Bayesian network meta-analysis. Journal of clinical neuroscience: official journal of the Neurosurgical Society of Australasia 70: 47-54.

264. Guo C, Xiang G, Xie L, Liu Z, Zhang X, et al. (2020) Effects of Tai Chi training on the physical and mental health status in patients with chronic obstructive pulmonary disease: a systematic review and metaanalysis. Journal of thoracic disease 12(3): 504-521.

265. Klein PJ, Baumgarden J, Schneider R (2019) Qigong and Tai Chi as Therapeutic Exercise: Survey of Systematic Reviews and MetaAnalyses Addressing Physical Health Conditions. Alternative therapies in health and medicine 25(5): 48-53.

266. Pölönen P, Lappi O, Tervaniemi M (2019) Effect of Meditative Movement on Affect and Flow in Qigong Practitioners. Frontiers in psychology 10: 2375.

267. Bao GC, Dillon J, Jannat-Khah D, Besada M, Marianova A, Mathewos, et al. (2019) Tai chi for enhanced inpatient mobilization: A feasibility study. Complementary therapies in medicine 46: 109-115.

268. Chen X, Cui J, Li R, Norton R, Park J, et al. (2019) Dao Yin (a.k.a. Qigong): Origin, Development, Potential Mechanisms, and Clinical Applications. Evidence-based complementary and alternative medicine: eCAM, 2019: 3705120.

269. Sabe M, Sentissi O, Kaiser S (2019) Meditation-based mind-body therapies for negative symptoms of schizophrenia: Systematic review of randomized controlled trials and meta-analysis. Schizophrenia research212: 15-25.

270. Sharma P, Mahapatra A, Gupta R (2019) Meditation-induced psychosis: a narrative review and individual patient data analysis. Ir J Psychol Med p. 1-7. 
271. Liu Z, Chen T, Shen M, Li K, Ma C, et al. (2020) A Pilot Study on the Effects of Yi Jin Jing Exercise for Older Men with Benign Prostatic Hyperplasia. Journal of aging and physical activity p. 1-6.

272. ChenJM, Wang ZY, Chen YJ, NiJ (2020) The Application of Eight-Segment Pulmonary Rehabilitation Exercise in People with Coronavirus Disease 2019. Frontiers in physiology 11: 646.

273. Guo C, Xiang G, Xie L, Liu Z, Zhang X, et al. (2020) Effects of Tai Chi training on the physical and mental health status in patients with chronic obstructive pulmonary disease: a systematic review and metaanalysis. Journal of thoracic disease 12(3): 504-521.

274. Tong H, Liu Y, Zhu Y, Zhang B, Hu J (2019) The therapeutic effects of qigong in patients with chronic obstructive pulmonary disease in the stable stage: a meta-analysis. BMC complementary and alternative medicine 19(1): 239

275. Yu F, Xin M, Liu N, Huang N, Lu J (2019) The Qigong Wuqinxi for chronic obstructive pulmonary disease: Protocol for a systematic review and meta-analysis. Medicine 98(30): e16633.

276. Zhang S, Zhu Q, Zhan C, Cheng W, Mingfang X, et al. (2020) Acupressure therapy and Liu Zi Jue Qigong for pulmonary function and quality of life in patients with severe novel coronavirus pneumonia (COVID-19): a study protocol for a randomized controlled trial. Trials 21(1): 751

277. Chen CH, Hung KS, Chung YC, Yeh ML (2019) Mind-body interactive qigong improves physical and mental aspects of quality of life in inpatients with stroke: A randomized control study. European journal of cardiovascular nursing: journal of the Working Group on Cardiovascular Nursing of the European Society of Cardiology 18(8): 658-666.

278. Gouw V, Jiang Y, Seah B, He H, Hong J, Wang W (2019) Effectiveness of internal Qigong on quality of life, depressive symptoms and selfefficacy among community-dwelling older adults with chronic disease: A systematic review and meta-analysis. International journal of nursing studies 99: 103378.

279. Jin X, Wang L, Liu S, Zhu L, Loprinzi PD, Fan X (2019) The Impact of Mind-body Exercises on Motor Function, Depressive Symptoms, and Quality of Life in Parkinson's Disease: A Systematic Review and Metaanalysis. International journal of environmental research and public health 17(1): 31 .

280. Lin WF, Zhong MF, Zhou QH, Zhang YR, Wang H, et al. (2019) Efficacy of complementary and integrative medicine on health-related quality of life in cancer patients: a systematic review and meta-analysis. Cancer management and research 11: 6663-6680.

281. Marks R (2019) Qigong and Musculoskeletal Pain. Current rheumatology reports 21(11): 59.

282. Sabe M, Sentissi 0, Kaiser S (2019) Meditation-based mind-body therapies for negative symptoms of schizophrenia: Systematic review of randomized controlled trials and meta-analysis. Schizophrenia research 212: 15-25.

283. Lane AE (2020) Practitioner Review: Effective management of functional difficulties associated with sensory symptoms in children and adolescents. Journal of child psychology and psychiatry, and allied disciplines 61(9): 943-958.

284. Dong X, Zhang R, Guo Y, Chen L, Liu Y, et al. (2020) The efficacy of Qigong exercises for post-stroke mental disorders and sleep disorders: Protocol for a systematic review and meta-analysis. Medicine 99(34): e21784.

285. Kim TY, Kim JH (2020) High school baseball players' experiences with static qigong training: A qualitative approach. Complementary therapies in clinical practice 39: 101158

286. Bischoff LL, Otto AK, Hold C, Wollesen B (2019) The effect of physical activity interventions on occupational stress for health personnel: A systematic review. International journal of nursing studies 97: 94-104.
287. Chen CH, Hung KS, Chung YC, Yeh ML (2019) Mind-body interactive qigong improves physical and mental aspects of quality of life in inpatients with stroke: A randomized control study. European journal of cardiovascular nursing: journal of the Working Group on Cardiovascular Nursing of the European Society of Cardiology 18(8): 658-666.

288. Dong X, Zhang R, Guo Y, Chen L, Liu Y, Lu Y (2020) The efficacy of Qigong exercises for post-stroke mental disorders and sleep disorders: Protocol for a systematic review and meta-analysis. Medicine 99(34): e21784.

289. Wang C, Yu L, Yang J, Wang RW, Zheng YN, et al. (2020) Effectiveness of LiuZiJue Qigong versus traditional core stability training for poststroke patients complicated with abnormal trunk postural control: study protocol for a single-center randomized controlled trial. Trials 21(1): 254.

290. Zheng Y, Zhang Y, Li H, Qiao L, Fu W, et al. (2020) Comparative effect of Liuzijue Qigong and conventional respiratory training on trunk control ability and respiratory muscle function in patients at an early recovery stage from stroke: a randomized controlled trial. Archives of physical medicine and rehabilitation S0003-9993(20): 30509-30518.

291. Liu F, Cui J, Liu X, Chen KW, Chen X, Li R (2020) The effect of tai chi and Qigong exercise on depression and anxiety of individuals with substance use disorders: a systematic review and meta-analysis. BMC complementary medicine and therapies 20(1): 161.

292. Tang J, Huang W, Chen X, Lin Q, Wang T, et al. (2019) Liuzijue Qigong: A Voice Training Method For Unilateral Vocal Fold Paralysis Patients. The Annals of otology, rhinology, and laryngology 128(7): 654-661.

293. Bao GC (2020) The idealist and pragmatist view of qi in tai chi and qigong: A narrative commentary and review. Journal of integrative medicine S2095-4964(20): 30060-300601.

294. Beissner F (2020) Therapeutic Sensations: A New Unifying Concept. Evidence-based complementary and alternative medicine: eCAM 2020: 7630190 .

295. Connor MH, Connor CA, Eickhoff J, Schwartz GE (2020) Prospective Empirical Test Suite for Energy Practitioners. Explore (New York, N.Y.) S1550-8307(20): 30228-30217.

296. Harvie A, Steel A, Wardle J (2019) Traditional Chinese Medicine SelfCare and Lifestyle Medicine Outside of Asia: A Systematic Literature Review. Journal of alternative and complementary medicine (New York, N.Y.) 25(8): 789-808.

297. Ibañez GE, Algarin A, Taskin T (2019) Letter Response: Yoga, Tai Chi, Qigong, and Health Disparities. American journal of public health 109(9): e5.

298. Matos LC, Machado J, Greten HJ, Monteiro FJ (2019) Changes of skin electrical potential in acupoints from Ren Mai and Du Mai conduits during Qigong practice: Documentation of a clinical phenomenon. Journal of bodywork and movement therapies 23(4): 713-720.

299. Toneti BF, Barbosa R, Mano LY, Sawada LO, Oliveira IG, et al. (2020) Benefits of Qigong as an integrative and complementary practice for health: a systematic review. Revista latino-americana de Enfermagem 28: e3317.

300. Zhang J, Su Q, Loudon WG, Lee KL, Luo J, et al. (2019) Breathing Signature as Vitality Score Index Created by Exercises of Qigong: Implications of Artificial Intelligence Tools Used in Traditional Chinese Medicine. Journal of functional morphology and kinesiology 4(4): 71.

301. Zhang YP, Hu RX, Han M, Lai BY, Liang SB, et al. (2020) Evidence base of clinical studies on Qi Gong: A bibliometric analysis. Complement Ther Med 50: 102392.

302. PubMed.gov. (2021) National library of medicine. National center for biotechnology information, National institute of health.

303. Europe PMC database (2021). 
304. Marks R (2017) Qigong exercise and arthritis. Medicines (Basel) 4(4): 71.

305. Brosseau L, Taki J, Desjardins B, Thevenot O, Fransen M, et al. (2017) The Ottawa panel clinical practice guidelines for the management of knee osteoarthritis. Part one: introduction, and mind-body exercise programs. Clin Rehabil 31(5): 582-595.

306. Brosseau L, Taki J, Desjardins B, Thevenot O, Fransen M, et al. (2017) The Ottawa panel clinical practice guidelines for the management of knee osteoarthritis. Part two: strengthening exercise programs. Clin Rehabil 31(5): 596-611.

307. Brosseau L, Wells GA, Pugh AG, Smith CA, Rahman P, et al. (2016) Ottawa Panel evidence-based clinical practice guidelines for therapeutic exercise in the management of hip osteoarthritis. Clin Rehabil 30(10): 935-946.

308. Brosseau L, Thevenot O, MacKiddie O, Taki J, Wells GA, et al. (2018) The Ottawa Panel guidelines on programmes involving therapeutic exercise for the management of hand osteoarthritis. Clin Rehabil 32(11): 1449-1471

309. Li Y, Wang K, Wang L, Chang T, Zhang S, et al. (2019) Biomechanical analysis of the meniscus and cartilage of the knee during a typical Tai Chi movement-brush-knee and twist-step. Math Biosci Eng 16(2): 898-908

310. You Y, Liu J, Tang M, Wang D, Ma X (2021) Effects of Tai Chi exercise on improving walking function and posture control in elderly patients with knee osteoarthritis: A systematic review and meta-analysis. Medicine (Baltimore) 100(16): e25655.

311. Zeng ZP, Liu YB, Fang J, Liu Y, Luo J, et al. (2020) Effects of Baduanjin exercise for knee osteoarthritis: A systematic review and metaanalysis. Complement Ther Med 48: 102279.

312. Li R, Chen H, Feng J, Xiao Y, Zhang H, et al. (2020) Effectiveness of traditional chinese exercise for symptoms of knee osteoarthritis: a systematic review and meta-analysis of randomized controlled trials. Int J Environ Res Public Health 17(21): 7873.

313. Xiao C, Zhuang Y, Kang Y (2020) Effects of Wu Qin xi Qigong exercise on physical functioning in elderly people with knee osteoarthritis: A randomized controlled trial. Geriatr Gerontol Int 20(10): 899-903.

\section{ISSN: 2574-1241}

DOI: $10.26717 /$ BJSTR.2021.37.006051

Robert W McGee. Biomed J Sci \& Tech Res

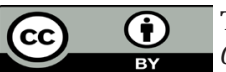

This work is licensed under Creative

Commons Attribution 4.0 License

Submission Link: https://biomedres.us/submit-manuscript.php
314. Lee HJ, Park HJ, Chae Y, Kim SY, Kim SN, et al. (2009) Tai Chi Qigong for the quality of life of patients with knee osteoarthritis: a pilot, randomized, waiting list-controlled trial. Clin Rehabil 23(6): 504-511.

315. Duan J, Wang K, Chang T, Wang L, Zhang S, et al. (2019) Tai Chi Is Safe and Effective for the Hip Joint: A Biomechanical Perspective. J Aging Phys Act 28(3): 415-425.

316. Zou L, Wang C, Chen K, Shu Y, Chen X, et al. (2017) The Effect of Taichi Practice on Attenuating Bone Mineral Density Loss: A Systematic Review and Meta-Analysis of Randomized Controlled Trials. Int J Environ Res Public Health 14(9): 1000.

317. Escalante Y, Saavedra JM, García Hermoso A, Silva AJ, Barbosa TM (2010) Physical exercise and reduction of pain in adults with lower limb osteoarthritis: a systematic review. J Back Musculoskelet Rehabil 23(4): $175-186$

318. Uhlig T (2012) Tai Chi and yoga as complementary therapies in rheumatologic conditions. Best Pract Res Clin Rheumatol 26(3): 387398.

319. Xie Y, Guo F, Lu Y, Guo Y, Wei G, et al. (2018) A 12-week Baduanjin Qigong exercise improves symptoms of ankylosing spondylitis: A randomized controlled trial. Complement Ther Clin Pract 36: 113-119.

320. Lee MS, Pittler MH, Ernst E (2007) Tai chi for rheumatoid arthritis: systematic review. Rheumatology (Oxford) 46(11): 1648-1651.

321. Imoto AM, Amorim FF, Palma H, Lombardi Júnior I, Salomon AL, et al. (2021) Evidence for the efficacy of Tai Chi for treating rheumatoid arthritis: an overview of systematic reviews. Sao Paulo Med J 139(2): 91-97.

322. Wang C (2008) Tai Chi improves pain and functional status in adults with rheumatoid arthritis: results of a pilot single-blinded randomized controlled trial. Medicine and Sport Science 52: 218-229.

323. Uhlig T, Fongen C, Steen E, Christie A, Ødegård S (2010) Exploring Tai Chi in rheumatoid arthritis: a quantitative and qualitative study. BMC Musculoskeletal Disorders 11: 43

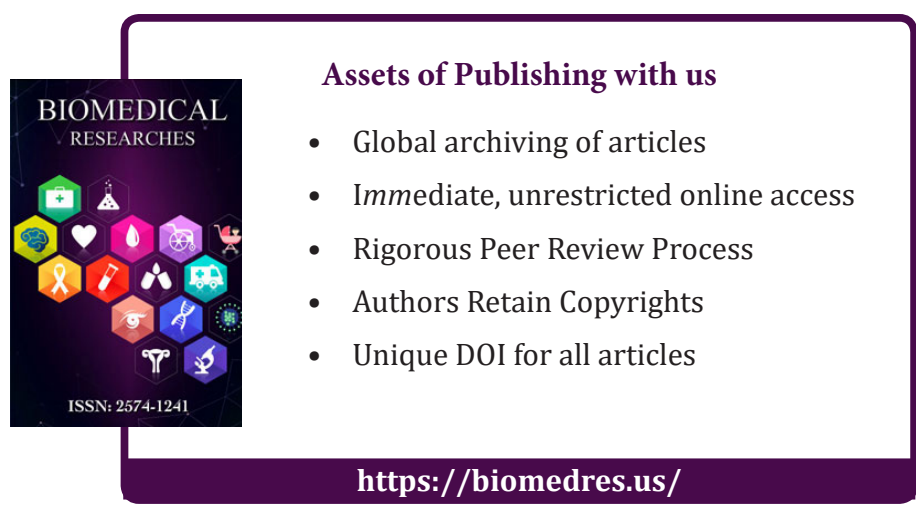

